\title{
Application of Formalization Method in Construction ZigBee Technology and RFID System in Internet of Things
}

\author{
Chunlin Kuang, Weiling Li \\ College of Information Technology \\ Luoyang Normal University \\ Henan LuoYang, China \\ kuangchunlinls@163.com
}

\begin{abstract}
RFID technology is the use of radio frequency signal through space coupling to achieve non-contact transmission of information through the transmission of information to automatically identify the purpose of technology. ZigBee protocol stack structure is composed of a set of the layer of the die block. Each layer is a layer above the execution of a set of specific services: data entity and data transmission service, management entity to provide all of the services. The paper presents application of formalization method in construction ZigBee technology and RFID system in Internet of things. Formal method is an important means to improve the system safety and reliability. BCCS model overcomes the shortcoming of the traditional method separates the information system of each facet.
\end{abstract}

Keywords- RFID; ZigBee; Formalization method; BCCS; Internet of things

\section{INTRODUCTION}

With the wide application of computers and technology, especially the application of computer system in high-tech fields, safety and reliability of the system has become a very important question. Formal method is an important means to improve the system safety and reliability. In fact, in various stages of development trend of formal methods gradually integrated into the software development process, from the demand analysis, function description (description), (Architecture / design, programming algorithm), testing and maintenance. Formal method is a method using mathematic and logic method to describe and verify the software. It can be divided into formal description and based on the formal description of formal verification based on the formal verification.

The concept of things is based on "the concept of the Internet", its extension and expansion of the client to any goods and goods between a network concept, the exchange of information and communications [1]. Its definition is: through the radio frequency identification (RFID), infrared sensors, GPS, laser scanners and other information sensing device, as agreed in the agreement, can make anything connected to the Internet, the exchange of information and communication, to realize intelligent identification, location, tracking, monitoring and management of a network of concepts.
ZigBee protocol stack length on average only Bluetooth or other IEEE 802.11 1/4, this simplification to the low cost, interactive and maintainability is very important. ZigBee technology provides data integrity and authentication function, provides three levels of security, flexibility to determine its safety properties, network security can be effectively guaranteed.

Radio frequency identification system should include at least the following two parts, one is the reader, and two is the electronic label (or RF card, transponder, this paper referred to as the electronic tag). Also should include the antenna, the host etc.. The RFID system in the specific application process, according to the application purpose and different application environment, system composition will be different.

Formal description (also called formal specification) is the use of formal language, grammar, which has rigorously defined semantics of language to describe the system and its requirements and characteristics. Tell from the description, formal method is a kind of system or program description, on the other hand is a description of the nature. These descriptions can be achieved in one or more languages, commonly used with $\mathrm{Z}$ language, VDM. B language for sequential systems, process algebra for concurrent systems (CSP, CCS, two calculus), state diagram (state charts), temporal logic, I/O automata, and other such as RAISE, LOTOS language and so on. The paper presents application of formalization method in construction ZigBee technology and RFID system in Internet of things.

\section{USING FORMALIZATION METHOD TO BUILD RFID SYSTEM IN INTERNET OF THINGS}

Mathematical theory and the description method based on different, thus also differ in thousands of ways, but they have a common characteristic, namely each description language are composed of basic component description and structural components described in two parts. The former is used to describe the basic (atomic) component, the latter the basic components combined into description list. Structural component is a formal description of research and design key point, but also the main basis to measure the quality of the description language.

The perceptual layer is to solve the problems of obtaining the human world and the physical world data. It 
begins by sensor, digital cameras and other equipment, collection of the external physical world data, and then through the RFID, bar code, the industrial field bus, Bluetooth, infrared and short distance transmission technology to transmit data. The key technology of perception layer which includes the detection technology, the short distance wireless communication technology.

Only the readable and writable label system only needs a programmer. The programmer is to write data to the device label. The programmer to write the offline data in general (OFF-LINE) completed also is in advance of the label to write data, when the application starts directly to the label adhesion in the identified projects. There is also some RFID application system, the write data is online (ON-LINE) completed, especially as interactive portable data files in a production environment to treatment. The antenna is a data transmitting, receiving device transmission between tags and reader.

The communication channel is established between the wireless signal reader layer and the label layer in RFID system. Process of space in the transmission channels can be divided into 3 kinds of event model: data exchange is objective; timing is the data exchange realization way; the energy is the foundation to realize the timing [2]. Reading and writing device layer using antenna electromagnetic signal, when the label in the reader's scope of work, the label instruction data and energy from the electromagnetic signal, the label and the data in the form of electromagnetic signals back to the reader, read operation; of course, the reader can also be changed to write stored in RFID tags data.

This paper presents two sets of inference rules, a description of the BCCS environment is to be described, we expressed as the following formula.

\section{$B C C S \vdash B C C S E$}

Clock, power of most RFID are AC signal shaping using antenna are obtained by changing the AC signal, so the harmonic amplitude, symmetry, frequency and other parameters can be implemented clock - power fault attack. With the help of RFID contact test equipment in the direct digital synthesis technology of AC signal, is very easy to have the clock - power fault attack the required waveform. RFID products in order to effectively resist the clock fault attack, besides using the clock detector, more important is to strictly limit the design of the RFID frequency range, carrier frequency harmonic quality factor, symmetry index.

RFID detection technology and specification: for example for different industrial applications of RFID tags and related products, physical properties and performance consistency detection technology and specification, between the reader and the tag air interface conformance testing technology and specification, and system solutions and comprehensive detection technology and standard.

Bidirectional authentication using PKI technology, the RFID subsystem, and it is set the read and write permissions for the sensitive area, business data encrypt, because J2ME MIDP 2 increase of HTTPS, SSL/TLS and X.509 PKI support, between the mobile phone and mobile payment platform adopts the bidirectional authentication of end to end encryption based on, to improve the security of mobile payment. The choice of China Financial
Certification Center (CFCA) as their CA, and it is digital certificate format to comply with X.509 V3 standard.

Design of EPC middleware enterprise information interaction network combines the characteristics of traditional middleware, the remote object by calling CORBAR and RMI based on J2EE technology, to achieve the greatest degree of distributed software architecture. Later in the middleware, in the form of SOA Web Service release in the enterprise service bus, convenient client calls. At the same time, the uses of J2EE EJB technology clear norms transaction operations and transaction management, and J2EE container corresponding to the transaction management middleware.

Bruns proposed unified value syntax, the value can be a tuple, and set and sequence can be expressed in the language to describe. The precise meaning of the language can be used to give the operational semantics [3]. In the Bruns report, processing of data in the system is a language of the statute, the language to meet the most basic feature model based method, such as the collection and predicate. In the BCCS model, using J-R Abrial B language is presented to describe the value system. Five abstract syntax of B class definitions in one (Table 1), we introduce the five basic grammar class as a BCCS language.

TABLE I. BCCS LANGUAGE

\begin{tabular}{|c|c|}
\hline Syntactic class & Definition \\
\hline$S P E C$ & SPEC \\
\hline PRED & $\begin{array}{l}P R E D \wedge P R E D \\
P R E D \Rightarrow P R E D \\
\neg P R E D \\
\forall V A R \cdot P R E D \\
{[V A R:=E X P] P R E D} \\
E X P=E X P \\
E X P \in S E T\end{array}$ \\
\hline$E X P$ & $\begin{array}{l}V A R \\
{[V A R:=E X P] E X P} \\
E X P, E X P \\
\text { choice(SET) } \\
\text { SET }\end{array}$ \\
\hline$V A R$ & $\begin{array}{l}\text { Indentifier } \\
V A R, V A R\end{array}$ \\
\hline SET & $\begin{array}{l}\text { SET } \times \text { SET } \\
\mathrm{P}(S E T) \\
\{V A R \mid P R E D\} \\
B I G\end{array}$ \\
\hline
\end{tabular}

SRFID technology is the use of radio frequency signal through space coupling (alternating magnetic field or electromagnetic field) to achieve non-contact transmission of information through the transmission of information to automatically identify the purpose of technology. In recent years, RFID technology is widely used in the field of logistics, the unique role is one of the important industries and application technology of it. RFID technology, it is to be recognized as the most promising that any object or 
person to mark the real world in the virtual world of network.

In this model, with an RFID tag card reader through the RFID (Reader) generates a Capturing event in door or gate, the event data is transmitted to the background information through middleware server, information server and then to find the matching context rule ID and choose a "notification service" (Notification, Service) needs to be pointed out is, to protect environment, safety information server sends a UIBS system to receive time and parameters to the RFID Privacy and security management Service) certification system.

Context is mainly for value expressions to define some of the more complex CCS. One that all abstract machine variables, also said the entire abstract machine, as is shown by equation 2 .

\section{$\int \hat{=}$ INITIALIZATION $\times$ INITIALIZATION}

Due to cost constraints, very limited storage space RFID label, and some are even only for unique identification. The RFID tag has some limitations in computing power and power consumption. At the same time tag does not have its own security ability enough, so will cause some illegal communicate with tags, or tampering, remove the label information. So the label of the security, integrity, availability, authenticity, and it is validity in the protection mechanism of the trusted enough to guarantee.

Tag and reader layer communicates through electromagnetic wave using radio frequency signal, without any physical contact and visible in the process of communication, the Internet of things perceived node layer and equipment generally exists in the open environment, the node energy and equipment, processing capabilities and communication range, not to the high strength encryption operation, the to provide flexibility and convenience at the same time to the application of data acquisition system, but also to make the message the lack of safety protection ability of the complex.

Non contact IC card RFID is important in the development of smart card milepost: it through magnetic coupling or microwave way to achieve non-contact transmission of energy and signal, so as to effectively solve the electrostatic breakdown, mechanical wear, easy to be polluted and wet environment influence of contact type intelligent card produced using mechanical electrical contacts, is considered to be an important alternative identification technology, transportation, logistics and other aspects of the bill [4]. There is no electrical contact node bare, compared to RFID and the contact type IC card products, in the aspect of safety of a certain improvement, but it does not change the smart card authentication algorithm and key security means and mode, and therefore did not solve the security problem in essence.

Middleware is a kind of message oriented, can accept applications from the end of the specified request, one or more of the reader an operation is initiated and receiving, processing to application software returns a result data of special software. Middleware in the application of RFID in addition to reliability and stability problems caused by various business scenarios, hardware interface, to shield the underlying hardware bring applicable standards, cooperation can also provide business information and management information for the upper application software layer, distributed, heterogeneous information environment. Main memory database middleware can be calculated according to one or more reader event filtering, aggregation and, abstracting the business logic information meaningful to the application software of business events from multiple clients, to meet the retrieval, publish / subscribe and control request.

Formal verification or search through inference to determine whether the system meets these requirements and has these characteristics. There is a close relationship between the formal verification and formal description, formal verification is to verify the existing applications (system), and whether to meet the requirements described (namely), one of the important problems which are a formal method to solve. Below we give the operational semantics of clipping value CCS, first give each process expression action set and free variables as shown in Table 2.

TABLE II. THE PROCESS EXPRESSION ACTION SET.

\begin{tabular}{|c|c|c|}
\hline $\begin{array}{l}\text { Process } \\
\text { expression }\end{array}$ & $L(e)$ & $F V(e)$ \\
\hline 0 & $\varnothing$ & $\varnothing$ \\
\hline$a\left(x_{1}, \mathrm{~K}, x_{2}\right)$ & $e^{e} L\left(e^{\prime}\right) \mathrm{Y}\{a\}$ & $F V\left(e^{\prime}\right)-\left\{x_{1}, \mathrm{~K}, x\right\}$ \\
\hline $\bar{a}\left(f_{1}, \mathrm{~K}, f_{2}\right) \cdot \dot{e}^{\prime}$ & $L\left(e^{\prime}\right) \mathrm{Y}\{\bar{a}\}$ & $F V(e) Y \underset{i}{Y}\left\{\underset{Y}{Y} F\left(f_{i}\right)\right\}$ \\
\hline$\tau . e^{\prime}$ & $L\left(e^{\prime}\right)$ & $F V\left(e^{\prime}\right)$ \\
\hline$e_{1}+e_{2}$ & $L\left(e_{1}\right) \mathrm{Y} L\left(e_{2}\right)$ & $F V\left(e_{1}\right) \mathrm{Y} F V\left(e_{2}\right)$ \\
\hline$e_{1} \mid e_{2}$ & $L\left(e_{1}\right) \mathrm{Y} L\left(e_{2}\right)$ & $F V\left(e_{1}\right) \mathrm{Y} F V\left(e_{2}\right)$ \\
\hline$e \backslash A$ & $L(e)-A-\bar{A}$ & $F V(e)$ \\
\hline$e[S]$ & $S(L(e))$ & $F V(e)$ \\
\hline $\begin{array}{l}\text { if } b \text { then } e_{1} \\
\text { else } e_{2}\end{array}$ & $L\left(e_{1}\right) \mathrm{Y} L\left(e_{2}\right)$ & $F V(b) Y F V\left(e_{1}\right) Y F V\left(e_{2}\right)$ \\
\hline
\end{tabular}

The context to describe the parameters, according to the definition in front of grammatical category, is composed of a B abstract machine,,, so in the grammar structure of BCCS parameters are more similar to the B method, rather than the value of CCS in the description of values, of course, if the value passing CCS parameters can be described well, we also you can use [5]. In addition, it also can be used to predicate formula of parameter constraint declaration. Such as is equation3.

$$
\operatorname{in}(x, y \mid x \in \mathrm{N} \wedge y \in \mathrm{N} \wedge x>y) . E
$$

Similarly, in the BCCS in addition to the parameters can describe the input action, parameters can describe process, as is the following formula4. 


$$
A(x, y \mid x \in \mathrm{N} \wedge y \in \mathrm{N} \wedge x>y)=E
$$

Value expression is used to describe the output action and process parameters, can be used to set variables, and represented, such as: is the legal process, prefix, where the parameters are value expression. And value expressions can also be used as the process parameters, such as the following formula 5 .

$$
\text { Polar }=\{\rho, \theta\} \subseteq \text { CONTEXT } T_{\text {POLARTOCART }}
$$

In this system, B data and operation do a structured definition, the most important is from the definition of B to extract the context, can some complex parameters defined in the system and the state through the context. Part CCS describes the evolution process in detail. We saw the B language and the value of CCS in BCCS have been well integrated.

In practical application of RFID, attached to the identified object (surface or internal) tag, only store some basic information about the object itself, the system cannot understand object state and change information between the environment via the tag, and RFID interference is poor and limited transmission distance (the distance generally less than 10m). The application of these shortcomings of RFID itself on its constitution to certain restrictions, the advantages of wireless sensor networks in the network and self perception of external information to just can make up for these disadvantages of RFID, this will make the RFID function to strengthen, expand the scope of application.

Block diagram of RFID system based on context aware system, the system is safe and reliable control by crawling the label and extraction of user information. First of all, the system structure of IS server system is context driven source, based on the context of events defined in advance good correlation between the specified service in a continuous topological tree. The IS server in the diagram represents information server, context processing and event data following filter, represented by the contextual rules in a continuous event element mapping. Continuous monitoring, it can perform data processing.

\section{CONSTRUCTION ZigBeE TEChNOLOGY BASED ON FORMALIZATION METHOD}

According to the $\mathrm{B}$ method in semantic concurrent increase in content, such as the original B method of operation (OPERATIONS) clause into the event (EVENTS) clause, increase the dynamic clause (DYNAMICS), the B abstract machine (AMN) is regarded as an interactive system. This Event B is similar to the commonly used Petri net to describe dynamic, concurrent process tool, but also inherits the $\mathrm{B}$ method in the functional data description, and its basic structure.

For the description of value passing CCS value, need to provide rich expression, mature universal grammar [6]. This includes practical structures, such as equality, function, relation, predicate, context; these have all been reflected in Bruns language. How to solve the said information system behavior description method based on frame model.

In sensor network based on ZigBee, the full function device as a Sink node, terminal nodes generally use cut function equipment to reduce the system cost and power consumption, improve the service life of the battery. Large capacity, short delay, node a single network can accommodate higher density. A ZigBee network can accommodate up to 254 from the equipment and 1 main equipment, a region can have 100 ZigBee network exist, and especially large-scale sensor array can meet the requirements.

Usually, the underlying FFD and RFD by MCU (micro controller) control, through the MCU cohort QSPI (serial peripheral interface) is connected with the ZigBee transceiver. The choice of MCU depends on whether the device as a still has jurisdiction of network layer in ZigBee FFD. Based on the RFD are usually controlled by a 8 bit MCU, but for FFD, according to its degree of complexity and network connection, the control unit can be $32 \mathrm{MCU}$. 8 bit, 16 bit or low-end.

ZigBee is a wireless communication protocol based on IEEE 802 and 15.4, it is a short distance, low power consumption protocol, used for small devices such as thermostats, lighting controller, ballast, environment detection sensor and medical equipment etc.. Based on a wireless personal area network (WPAN) ZigBee can hold up to 254 nodes, and a full function device, namely realizing two-way communication. Characteristics of low data rate of ZigBee technology and ZigBee technology small communication range of decisions for carrying small data volume of business.

The system of self-organization function without manual intervention, the network node can perceive the presence of other nodes, and determine the connection, to form a structured network; and Zigbee self-healing function can be completed by adding or deleting a node, processing or change occurs in the node position. Node failure, so that the network can self repair, and the network topology are adjusted accordingly, without human intervention, so that the whole system can still work normally. Can say, wireless communication network has self-organization, self-healing ability.

BCCS model overcomes the shortcoming of the traditional method separates the information system of each facet [7]. In BCCS model, two important facets of information systems are unified. As can be seen, BCCS model using CCS and B language combining the ideas of value, using the B language extensions to CCS in the data and function is defined in terms of ability, and through the CCS extension B method in the clear ability of operational semantics and describe aspects of concurrent systems. This integration is the result of a more expressive formal development method, to describe the problem from more facets, and also provides a new attempt for the development of formal methods in system.

Based on the simple description of the system, we can understand the BCCS syntax clearly. Fig .1 shows the system state transitions.

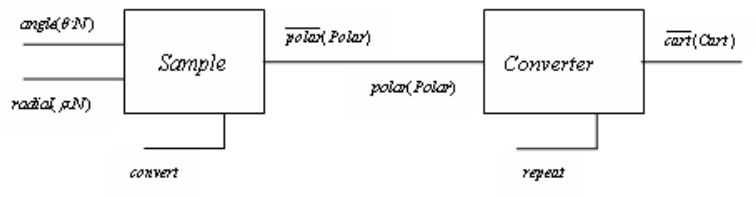

Figure 1. The status of BCCS system conversion. 
According to the traditional value of CCS on the process definition is this is legitimate, because it represents the same parameters (input, output), but in the mixed model in BCCS, a global variable is defined in B, another is a local variable declaration in the process, obviously they are not the same not so, says. Faced with this situation, in the BCCS model we use environment on the constant environment description, a global variable is described by $\mathrm{B}$, another is a local variable process, so that the two will not occur earlier, because the "conflict parameters caused by residual".

First give a constructor, to construct; using recursive method, from other smaller trees began to construct the environment. Constructor required object can only get. We define a process environment, the environment is constant and the parameter constant to part mapping they define process. Set and respectively by the previously defined syntax and depicted, these grammatical category is of some referential expressions, representing the object they portray.

\section{$\frac{B \vdash B E}{B \vdash C O N T E X T}$}

VDM is a kind of functional structural specification techniques, it describes each operation or function through the first-order predicate logic and established abstract data types, functions, the functions of the software system is described in the abstract level, completely get rid of the implementation details, so there is a big advantage is that provides great flexibility for the software, but also caused the VDM formal specification is too formal and often not easy to understand. VDM has failed to establish a set of description mechanism, can be a large-scale system is decomposed into many calculations and describe the relations between these operations.

Z-CSP is the absorption of other language from a semantic level in a language description, thus different expression systems in a language category. Describes the type of semantic CSP applications to the $\mathrm{Z}$ language, which is concurrent define a symbolic transition, interaction is similar to that of the CSP process, allowing direct uses $\mathrm{Z}$ to describe the operation. This method from the theoretical point of view, more meaningful, however, compared with the BCCS model, in the practical level, but not to $\mathrm{Z}$ or CSP have further excavation, there is a certain distance from the actual application.

Looking for a way, absorb and integrate other methods or the description of a language at the semantic level, which can express various aspects of language in a category. Describe the kinds of semantic CSP applications to the $\mathrm{Z}$ language, which is concurrent define a symbolic transition, interaction is similar to that of the CSP process, allowing direct uses $\mathrm{Z}$ to describe the operation.

To sum up, formal methods require formal description language support, formal description language is the basis of formal methods, the research work of this paper is how in the unified framework for concurrent, distributed systems formal description.
IV. APPLICATION OF FORMALIZATION METHOD IN CONSTRUCTION ZIGBEE TECHNOLOGY AND RFID SYSTEM IN INTERNET OF THINGS

Formal verification or search through inference to determine whether the system meets these requirements and has these characteristics. There is a close relationship between the formal verification and formal description, formal verification is to verify the existing applications (system), whether to meet the requirements described, one of the important problems which are formal methods to solve.

The definition of the BCCS syntax, operational semantics of BCCS is given, on this basis; we constructed a BCCS model [8]. In this model, can achieve the first objective, in addition, it allows the data structure is integrated into B defined context and CCS process. By B and CCS jointly organized a clear for complex large-scale concurrent or distributed system data and behavior, and this is described by the traditional method is not feasible. At the same time, the BCCS model also completed the theoretical basis and description language to achieve the second target, now need only one BCCS can realize the software environment.

ZigBee is a group of technical standards developed by IEEE approved 802.15.4 Wireless Standard Based on network, security and application software. The complete Zigbee protocol suite by the application of high standards, and it is convergence layer, network layer, data link layer and physical layer. Zigbee is based on IEEE802.15.4 and IEEE for low energy consumption, simple equipment to provide effective coverage is about $10 \sim 100 \mathrm{~m}$ in the low speed connection wireless interconnection standards. This standard defines the physical layer in Zig-bee protocol suite (PHY) and data link layer of the MAC sublayer.

A complete RFID system includes database server, tags and readers. Although the database itself and the reader to the server authentication has the security problem, but they have enough energy and computing resources, can use encryption algorithm to ensure the safety of traditional. Compared to RFID tag brings security and privacy problem is particularly prominent. Communication media between the RFID tag and reader's radio, is completely exposed to the attacker and other unauthorized reader, so the lack of confidentiality of information transmission.

BCCS model to ensure its own characteristics at the same time, also fully embodies the advantages of CCS method and B method of these two formal values [9]. We select the value passing CCS and B methods as hybrid objects, mainly because the CCS in the concise description of concurrent and distributed systems, B method in practical large order and usability of the system. So in a model of large concurrent or distributed systems is described as possible.

When the instance is in the ready state, he is free to accept the call from the client request, can be is transactional or non transactional. Timeout is removed or a call to the @PrePassivate method corresponding into not instantiate or passivation.

$$
B_{-} \text {Spec } \vdash B_{-} \text {Env }
$$




$$
\frac{B E \in \operatorname{ran}\left(\left(A M N_{1}^{\mathrm{M}} \mathrm{YK} \mathrm{Y} A M N_{n}^{\mathrm{M}}\right) \mathrm{o} \varepsilon_{0}\right)}{B_{-} A M N_{1} \mathrm{~K} B_{-} A M N_{2} \vdash B E}
$$

$$
v p_{-} C C S_{-} \text {Agents } \vdash A_{-} E n v
$$

$$
\frac{<<B E, B E^{\prime}, A E>, F>\stackrel{\alpha}{\longrightarrow}<<Z E, T, A E>, F^{\prime}>}{<<B E, B E^{\prime}, A E>, G+F>\stackrel{\alpha}{\longrightarrow}<<B E, T, A E>, F^{\prime}>}
$$

$$
\begin{aligned}
& \ll B E, T, A E>, F>\stackrel{\alpha}{\longrightarrow}<B E, T^{\prime}, A E>, F^{\prime}> \\
& \frac{T=j \operatorname{join}\left(B E^{\prime}, B E^{\prime}\right)}{\ll B E, B E^{\prime}, A E>, F>\stackrel{\alpha}{\longrightarrow}<<B E, T^{\prime}, A E>, F^{\prime}>}
\end{aligned}
$$

The session Bean is used for business process modeling. In the EJB3.0 specification, the SFSB services business process can be extended to multiple requests or transaction, therefore, need to save the state information for each customer. If there is a change in state session state occurs during a method call, the change must be reflected in subsequent calls to the same customer. In the EJB3.0 specification suggests that can complete the initialization of SFSB through the custom methods, and expose them in the business interface.

Especially the modular technology smooth B into the BCCS model, because there is no modular technology, any one model cannot be applied to large systems, because the BCCS model inherits the modular technology B, for their application to large system and create the possibility of it, as is shown by Fig .2.

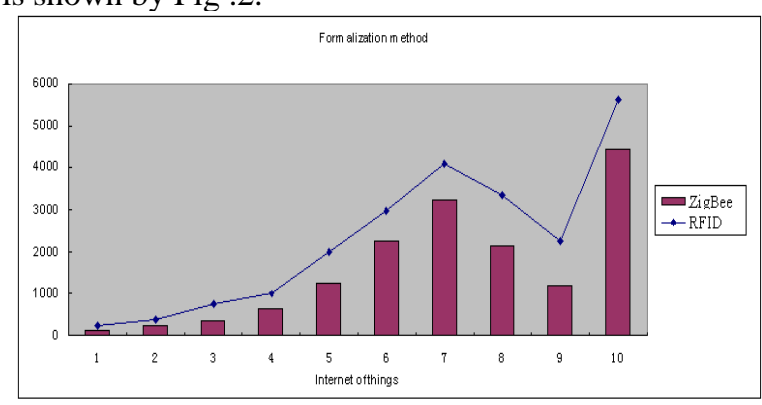

Figure 2. Comparison results of formalization method in construction ZigBee technology with RFID system.

The paper presents application of formalization method in construction ZigBee technology and RFID system in Internet of things. Preliminary research on the ZigBee network and its routing: discusses in detail the new ZigBee network establishment process and new equipment into the Zigl3ee network flow; the basic algorithm, routing, routing of ZigBee network routing discovery, routing cost algorithm is studied; based on S-MAC protocol, the construction of six node star network, and routing simulation.

Internet of things based on RFID technology system, tag and reader layer between the RF signal through implementation of the tag and reader layer of communication, the Internet to solve the read and write the communication between the device layer and the application layer. But because of the Internet of things all objects are connected to the Internet, but also it is applied to all walks of life and all aspects of daily life.

\section{SUMMARY}

BCCS model and many problems need to be further studied in it. First of all, in the BCCS model has not been fully realized refinement (Refinement) technique, the advantages of the BCCS model does not completely play B method. Secondly, the need to achieve a BCCS software environment, for formal verification based on BCCS model provides support tools. In a word, to let the BCCS model can run in the real computer, the BCCS model is not only a large concurrent or distributed system description language. These questions are what we should study the objectives of the future.

\section{REFERENCES}

[1] Axel van Lamsweerde, "Formal Specification:a Roadmap", the future of software engineering,2000

[2] Ming-Cheng Lee, Chi-Chung Lee, "Stable Tag Identification in Mobile RFID Systems”, JCIT, Vol. 7, No. 7, pp. 312 322, 2012.

[3] Zhou Shen grong, Zheng Guo liang, "Comparison of Z and VDM with B”, Computer Science, 2002, 29(10), 136-138.

[4] Yan Wang, "The Development of Wireless Personnel Positioning in Internet of Things Based on ZigBee and Sensors", JDCTA, Vol. 6, No. 12, pp. $47 \sim 54,2012$.

[5] Ho Sun Yoon, Heung Youl Youm, "An Anonymous Search Protocol for RFID Systems”, JCIT, Vol. 6, No. 8, pp. $44 \sim 50$, 2011.

[6] Jin-ling Zhang, Sheng Zhang, Ying-bo Guo, Xiong-zhi Zhu, Feixue Meng, "A ECG And Oxygen Saturation Monitoring System Based On ZigBee Mesh Network", JDCTA, Vol. 7, No. 9, pp. 124 $\sim$ 132, 2013.

[7] M.Butler, M.Leuschel, "Combining CSP and B for Specification and Property Verification", Proceedings of Formal Methods, 2005(LNCS 3582), 221-236.

[8] Jie Wu, Dong Wang, Huanye Sheng, “Architecting a Configurable and Reconfigurable RFID Infrastructure", JCIT, Vol. 6, No. 6, pp. $162 \sim 175,2011$.

[9] Michael J. Butler, “csp2B: A Practical Approach to Combining CSP and B”, Formal Asp. Comput, 12(3), 2000, 182-198 